\title{
POPULATION SYNTHESIS IN A UNIVERSE OF INTERACTING GALAXIES
}

\author{
GUSTAVO A. BRUZUAL \\ Centro de Investigaciones de Astronomía (C.I.D.A.) \\ Apartado Postal 264, Mérida, Venezuela
}

\section{Introduction}

The traditional view in population synthesis and evolutionary synthesis models assumes that galaxies can be considered closed systems. Thus, the evolution of the stellar population in galaxies which do not interact with the environment (including other galaxies) is described by the so called Pure Luminosity Evolution (PLE) model. As we have heard several times during this conference (and before), galaxies do interact and very few galaxies are expected to evolve passively. Early-type galaxies seem to behave closer to the PLE model than later types, but $\mathrm{E}$ galaxies in the bright phase predicted by this model at the galaxy formation epoch have not been observed. Most likely $\mathrm{E}$ galaxies do not form all their stars in short lived initial bursts (several talks in this conference). Number, size, and luminosity evolution is required to understand late-type and Irr galaxies. The PLE model is unable to reproduce the number counts, and color and redshift distributions of the galaxies in the HDF (Pozzetti et al. 1997).

Under these circumstances, we may ask ourselves: are population synthesis models still useful? I think that the answer is yes. In this conference we have seen already several applications of population synthesis models to interacting galaxies. Population synthesis models provide tools to understand the properties (colors, luminosity, evolutionary age, initial mass function, star formation rate, etc.) of the dominant population in star clusters and galaxies. The models also allow us to predict the effects of nonpassive events (mergers, starbursts) in the evolution of these systems. In this paper I will show some results from the Bruzual \& Charlot (1997, BC97 hereafter) evolutionary models suitable to study interacting galaxies. I make no attempt to review here work by other authors, notably Arimoto and collaborators, Bressan, Chiosi and collaborators, Buzzoni and collabo- 
rators, Fritze-v. Alvensleben and collaborators, Leitherer and collaborators, Rocca-Volmerange and collaborators, Vazdekis and collaborators, Worthey and collaborators. The reader is referred to various papers in this volume which illustrate the use of different sets of evolutionary models.

\section{Population Synthesis Models}

BC97 have extended the Bruzual \& Charlot (1993, BC93 hereafter) evolutionary population synthesis models to provide the evolution in time of the spectrophotometric properties of SSPs for a wide range of stellar metallicity. The BC97 models are based on the stellar evolutionary tracks computed by Alongi et al. (1993), Bressan et al. (1993), Fagotto et al. (1994a, b, c), and Girardi et al. (1996), which use the radiative opacities of Iglesias et al. (1992). This library includes tracks for stars with initial chemical composition $Z=0.0001,0.0004,0.004,0.008,0.02,0.05$, and 0.10 , with $Y=2.5 Z+0.23$, and initial mass $0.6 \leq m / M_{\odot} \leq 120$ for all metallicities, except $Z=0.0001\left(0.6 \leq m / M_{\odot} \leq 100\right)$ and $Z=0.1\left(0.6 \leq m / M_{\odot} \leq 9\right)$. This set of tracks will be referred to as the Padova or P-tracks hereafter. To allow for uncertainties in the stellar evolution theory, BC97 consider the evolutionary tracks computed by Schaller et al. (1992) $\left(m \geq 2 M_{\odot}\right)$ and Charbonnel et al. (1996) $\left(0.8 \leq m / M_{\odot}<2\right)$ for solar metallicity as an alternative to the P-tracks. In this case the abundances are $X=0.68$, $Y=0.30$, and $Z=0.02$, and the opacities are from Iglesias et al. (1992) (for $m \geq 2 M_{\odot}$ ) and Iglesias \& Rogers (1993) (for $0.8 \leq m / M_{\odot}<2$ ). This set of tracks will be referred to as the Geneva or G-tracks hereafter. For $Z_{\odot}=0.02$ both sets of tracks are normalized to the temperature, luminosity, and radius of the Sun at an age of $4.6 \mathrm{Gyr}$. The published tracks go through all phases of stellar evolution from the zero-age main sequence to the beginning of the thermally pulsing regime of the asymptotic giant branch (AGB, for low- and intermediate-mass stars) and core-carbon ignition (for massive stars), and include mild overshooting in the convective core of stars more massive than $1 M_{\odot}$ (Padova set) and $1.5 M_{\odot}$ (Geneva set). The Post-AGB evolutionary phases for low- and intermediate-mass stars were added to the tracks by BC97 from different sources (see BC97 for details).

The BC97 models use the library of synthetic stellar spectra compiled by Lejeune et al. (1997a,b, LCB97 hereafter) for all the metallicities listed above. This library consists of Kurucz (1995) spectra for the hotter stars (O-K), Bessell et al. (1989, 1991) and Fluks et al. (1994) spectra for M giants, and Allard \& Hauschildt (1995) spectra for M dwarfs. There are two versions of this atlas. One version (LCB97-O hereafter) contains the model spectra as published, but rebinned in a homogeneous fundamental 
parameter (effective temperature, gravity, metallicity) and wavelength scale (Lejeune et al. 1996). In the second version (LCB97-C hereafter), LCB97 corrected the original model spectra for systematic deviations that become apparent when color-temperature relations computed from the models are compared to empirical ones at $Z_{\odot}$. The corrections are especially important for the M star models. These semi-empirical blanketing corrections are defined for every wavelength, and should be a function of the fundamental model parameters: temperature, gravity, and $Z$. Due to the lack of calibration standards at various metallicities, LCB97 applied the corrections derived for $Z_{\odot}$ at all metallicities. Since the blanketing correction functions are multiplicative, the differential properties of the libraries are nearly conserved (see LCB97a,b for details). For instance, well known photometric differential metallicity indicators for F-G-K stars, such as the ultraviolet excesses, $\delta(U-B)$ in UBV photometry, and $\delta(C-M), \delta\left(C-T_{1}\right)$ in Washington photometry, are very well reproduced for dwarf and giants by both the original and the corrected versions of the Kurucz (1995) library (Lejeune \& Buser 1996). For $Z=Z_{\odot}$, BC97 also use an extended version of the Gunn \& Stryker (1983) atlas, assembled from mostly empirical stellar data (EGS atlas hereafter, see BC97 for details).

Regardless of the specific computational algorithm used, all evolutionary synthesis models depend on three adjustable parametric functions: (1) the stellar initial mass function, $f(m)$, or IMF; (2) the star formation rate, $\Psi(t)$, or SFR; and (3) the chemical enrichment law, $Z(t)$. For a given choice of $f(m), \Psi(t)$, and $Z(t)$, a particular set of evolutionary synthesis models provides: (1) Galaxy spectral energy distribution (SED) vs. time, $F_{\lambda}(\lambda, Z(t), t)$; (2) Galaxy colors and magnitude $v s$. time; (3) Line strength and other spectral indices $v s$. time.

Fig 1. shows the evolution in time of the $B-V$ and $V-K$ colors, and the $M / L_{\mathrm{V}}$ ratio predicted by BC97 for chemically homogeneous simple stellar populations (SSPs) of the indicated metallicity. In an SSP all the stars form at $t=0$ and evolve passively afterward. In all the examples shown in this paper I assume that stars form according to the Salpeter (1955) IMF in the range from $m_{\mathrm{L}}=0.1$ to $m_{\mathrm{U}}=125 M_{\odot}$. The total mass of the model galaxy is $1 M_{\odot}$.

From Fig 1 it is apparent that there is a uniform tendency for galaxies to become redder in $B-V$ as the metallicity increases from $Z=0.0004\left(\frac{1}{50} Z_{\odot}\right)$ to $Z=0.05\left(2.5 \times Z_{\odot}\right)$. The tendency reverses at the highest metallicity shown, $Z=0.10\left(5 \times Z_{\odot}\right)$. After $14 \mathrm{Gyr}$ this model becomes as blue as the lower $Z$ models. One reason for this behavior is the appearance of AGB-manqué stars at $Z=0.10$ (Greggio \& Renzini 1990). These stars skip the AGB phase and instead go through a long lived hot HB phase. However, this particular result should be taken with caution. The opacities, 


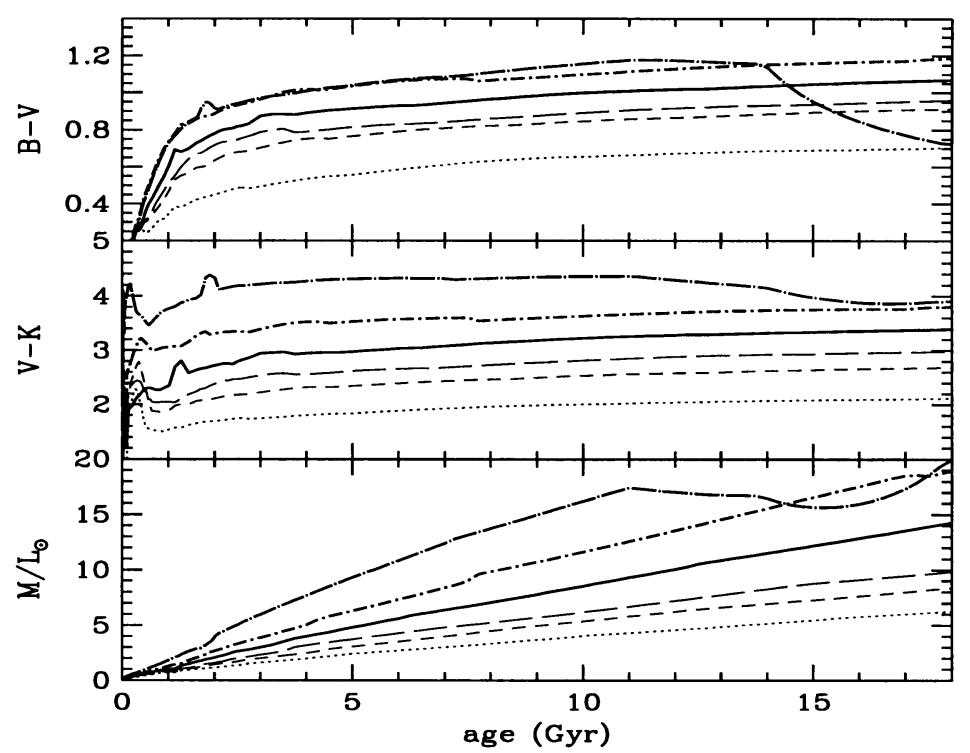

Figure 1. Evolution in time of the $B-V$, and $V-K$ colors, and the $M / L_{\mathrm{V}}$ ratio for BC97 models of different metallicities. Each line represents a different metallicity, as indicated in the middle panel in Fig 2. BC97 models constructed with the Padova tracks and the LCB97-C stellar library. See text for details.

and hence both the evolutionary tracks and the stellar model atmospheres, are quite uncertain at this high $Z$. There are very few, if any, examples of galactic stars which such a high $Z$. On the contrary, the $V-K$ color and the $M / L_{\mathrm{V}}$ ratio show the expected tendency with metallicity, i.e. $V-K$ becomes redder and $M / L_{\mathrm{V}}$ becomes higher with increasing $Z$. However, even in these two quantities there is a trend at $t>12 \mathrm{Gyr}$ for the $Z=0.10$ model to approach the $Z=0.05$ model.

Fig. 2 shows the evolution in time of the $\mathrm{Mg}_{\mathrm{b}}, \mathrm{H}_{\beta}$, and $\mathrm{Ca}$ spectral indices as defined by Worthey (1994) for the same BC97 SSP models shown in Fig. 1 Again, except for the $Z=0.10$ model, the models show the expected tendency with $Z$ and match the values computed by Worthey (1994). It should be remarked that the time behavior of the line strength indices at constant $Z$ is due to the change in the number of stars at different positions in the HR diagram produced by stellar evolution and is not related to chemical evolution. The indices change also in chemically homogeneous populations. The $\mathrm{H}_{\beta}$ index is less sensitive to the stellar metallicity than the $\mathrm{Mg}_{\mathrm{b}}$ and $\mathrm{Ca}$ index. Instead, the $\mathrm{H}_{\beta}$ index is high when there is a large fraction of MS A-type stars $(t<1)$ Gyr. The behavior of the 3 indices for $Z=0.10$ and $t>12 \mathrm{Gyr}$ is dominated by the presence of the hot 


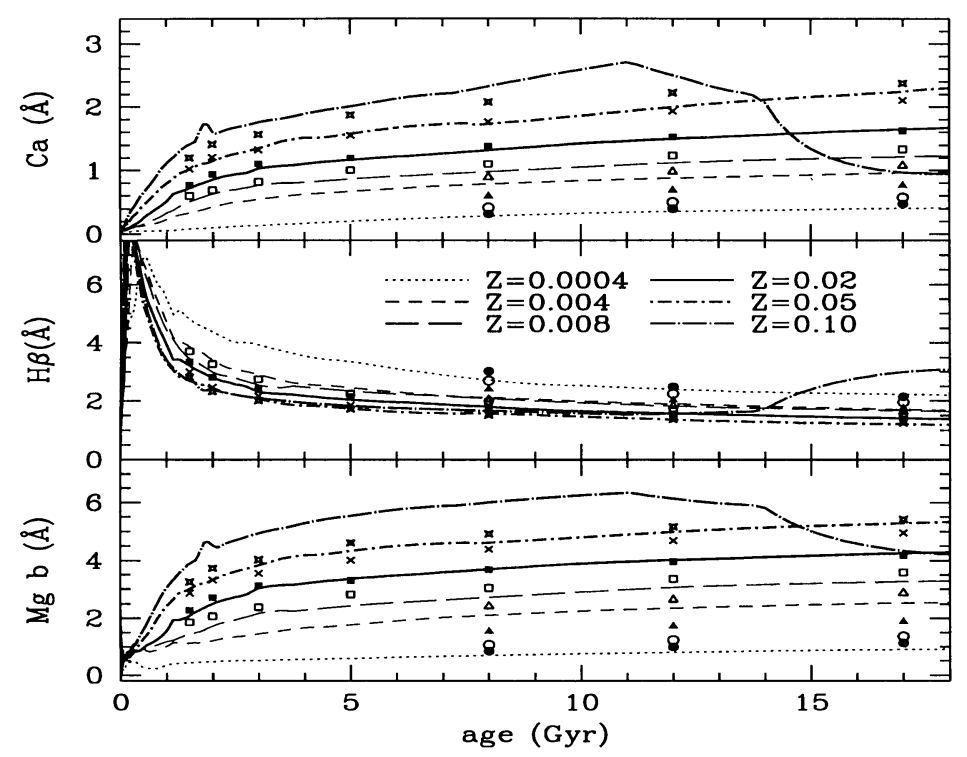

Figure 2. Evolution in time of the $\mathrm{Mg}_{\mathrm{b}}, \mathrm{H}_{\beta}$, and Ca spectral indices as defined by Worthey (1994) for the BC97 models shown in Fig. 1. The different symbols represent the values of the indices computed by Worthey for the same range in $Z$. Each line represents a different metallicity, as indicated in the middle panel. BC97 models constructed with the Padova tracks and the LCB97-C stellar library.

HB (AGB-manqué) stars. Again, this prediction is uncertain and should be taken with caution.

\section{Application to Interacting Galaxies}

As an example of the use of population synthesis to model galaxies with a more complex star formation history than traditionally assumed, I discuss here the case of galaxies described by the following functions $\Psi(t)$ and $Z(t)$.

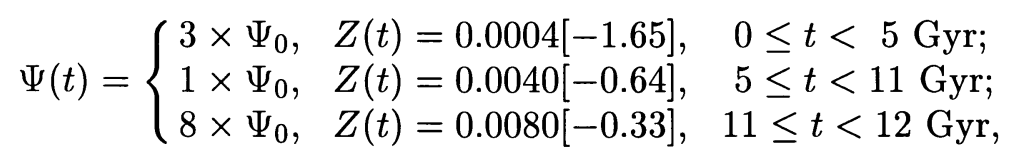

and

$$
\Psi(t)= \begin{cases}1 \times \Psi_{0}, & Z(t)=0.02[+0.09], \quad 0 \leq t<0.8 \mathrm{Gyr} \\ 1 \times \Psi_{0}, & Z(t)=0.05[+0.56], 0.8 \leq t<0.9 \mathrm{Gyr} \\ 1 \times \Psi_{0}, & Z(t)=0.10[+1.01], 0.9 \leq t<1.0 \mathrm{Gyr}\end{cases}
$$

The numbers inside the square brackets in (1) and (2) represent the value of $[\mathrm{Fe} / \mathrm{H}]$ for the corresponding $Z$ and $\Psi_{0}=1 M_{\odot} \mathrm{yr}^{-1}$. The complex nature of $\Psi(t)$ and $Z(t)$ may result from interactions with the environment 


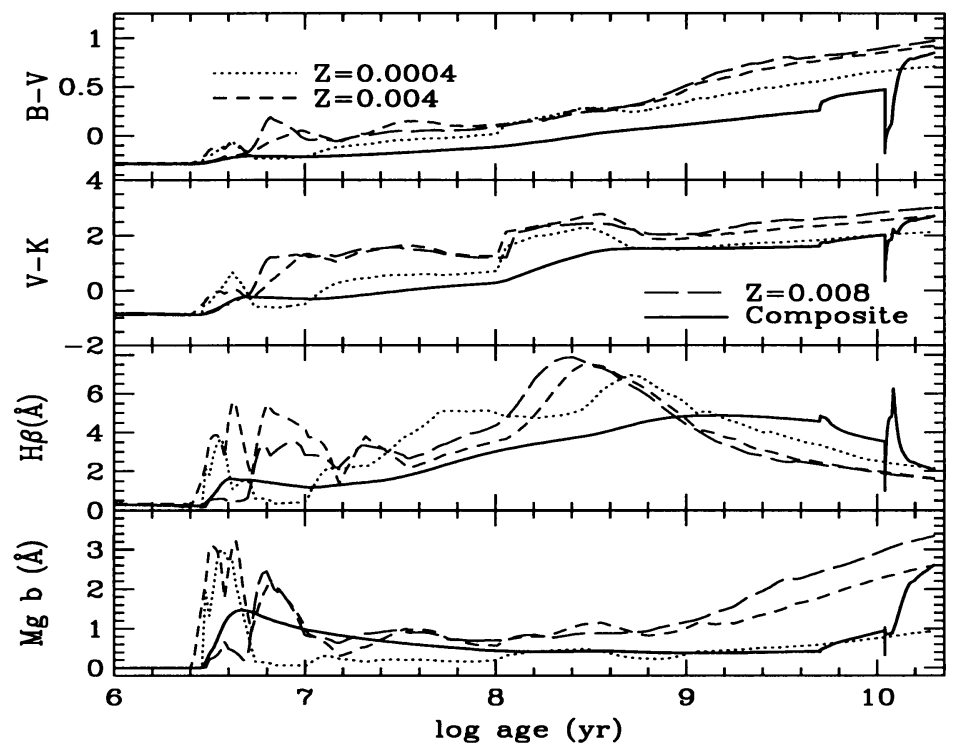

Figure 3. Evolution in time of the $B-V$ and $V-K$ colors and the $\mathrm{H}_{\beta}$ and $\mathrm{Mg}_{\mathrm{b}}$ spectral indices for the different SSPs used to build the composite population described by eq. (1). The heavy solid line represents the composite population. BC97 models constructed with the Padova tracks and the LCB97-C stellar library. Notice the logarithmic scale in the horizontal axis.

(other galaxies, IGM, dark matter) or from the galaxy formation process itself. The multiple bursts of star formation in (1) are typical of dIr galaxies characterized by episodic star formation (Smecker-Hane et al. 1995). The SFR (2) represents the history of star formation in E galaxies when metal enrichment above solar values during the last fifth of the initial burst is taken into account. Following BC93 we can compute the properties of the composite population described by (1) and (2) by convolving $\Psi(t)$ with the evolving spectrum of the SSPs for the corresponding $Z(t)$. For simplicity $f(m)$ is assumed to follow the Salpeter power law at all times.

The evolution in time of the B-V and V-K colors and the $\mathrm{H}_{\beta}$ and $\mathrm{Mg}_{b}$ spectral indices for the populations described by (1) and (2) are shown in Figs 3 and 4, respectively, together with the evolution of the same properties for the different SSPs used to build each composite population. A few things should be remarked from these figures. The properties of the composite population evolve more smoothly than for the SSPs due to the convolution with the SFR. The discontinuities in $\Psi(t)$ are also present in the integrated properties. The population becomes bluer following an increases in $\Psi(t)$. There is a delay in the corresponding increase of the $\mathrm{H}_{\beta}$ index lasting until the newly formed O-B stars leave the main sequence. Once passive 


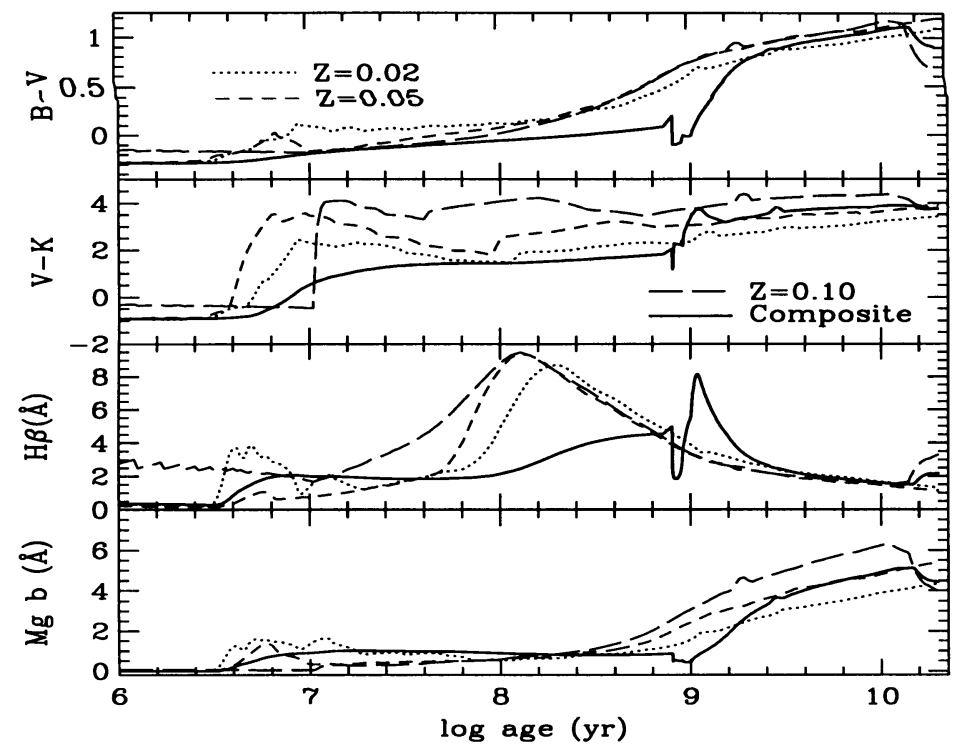

Figure 4. Evolution in time of the $B-V$ and $V-K$ colors and the $\mathrm{H}_{\beta}$ and $\mathrm{Mg}_{\mathrm{b}}$ spectral indices for the different SSPs used to build the composite population described by eq. (2). The heavy solid line represents the composite population. BC97 models constructed with the Padova tracks and the LCB97-C stellar library. Notice the logarithmic scale in the horizontal axis.

evolution sets in, the population reddens, $\mathrm{H}_{\beta}$ decreases, and $\mathrm{Mg}_{\mathrm{b}}$ increases. This tendency reverses in Fig 4 once the highest metallicity stars, $Z=0.10$ in (2) are formed, and the AGB-manqué stars appear: the integrated colors become slightly bluer, $\mathrm{H}_{\beta}$ increases, and $\mathrm{Mg}_{\mathrm{b}}$ decreases. As remarked above, this particular result should be taken with caution.

\section{Conclusions}

In this paper I have illustrated by means of two examples how to use simple stellar population synthesis models to study the observational properties of composite stellar populations subject to star formation laws plausible for interacting or forming galaxies of various kinds. Even though these models do not follow the dynamical processes involved in galaxy formation and interactions, they provide insight into the problem of spectral amd photometric evolution of interacting or forming galaxies. The results from population synthesis can be input into dynamical models to build selfconsistent models which include chemical and spectral evolution (see, for instance, several papers in this volume). 


\section{References}

Allard, F., \& Hauschildt, P.H. 1995, ApJ, 445, 433

Alongi, M., Bertelli, G., Bressan, A., Chiosi, C., Fagotto, F., Greggio, L., \& Nasi, E. 1993, A\&AS, 97, 851

Bessell, M.S., Brett, J., Scholtz, M., \& Wood, P. 1989, A\&AS, 77, 1

- 1991, A\&AS, 89, 335

Bressan, A., Fagotto, F., Bertelli, G., \& Chiosi, C. 1993, A\&AS, 100, 647

Bruzual A., G. \& Charlot, S. 1993, ApJ, 405, 538 (BC93) 1997, ApJ, in preparation (BC97)

Charbonnel C., Meynet G., Maeder A., \& Schaerer D., 1996, A\&AS, 115, 339

Fagotto, F., Bressan, A., Bertelli, G., \& Chiosi, C. 1994a, A\&AS, 100, 647

- 1994b, A\&AS, 104, 365 1994c, A\&AS, 105, 29

Fluks, M. et al. 1994, A\&AS, 105, 311

Girardi, L., Bressan, A., Chiosi, C., Bertelli, G., \& Nasi, E. 1996, A\&AS, 117, 113

Greggio, L., \& Renzini, A. 1990, ApJ, 364, 35

Gunn, J.E. \& Stryker, L.L. 1983, ApJS, 52, 121

Iglesias, C.A., Rogers, F.J., \& Wilson, B.G. 1992, ApJ, 397, 717

Iglesias, C.A., \& Rogers, F.J. 1993, ApJ, 412, 752

Kurucz, R. 1995, private communication

Lejeune, T., Buser, R. 1996, Baltic Astronomy, 5, 399

Lejeune, T., Cuisinier, F., \& Buser, R. 1996, in "A Data Base for Galaxy Evolution Modeling", eds. C. Leitherer et al., PASP, 108, 996 1997a, A\&AS, 125, 229

$1997 \mathrm{~b}, \mathrm{~A} \& \mathrm{~A}$, in preparation (LCB97b)

Pozzetti, L., Madau, P., Zamorani, G., Ferguson, H.C., \& Bruzual A., G. 1997, MNRAS, in press

Salpeter, E.E. 1955, ApJ, 121, 161

Schaller G., Schaerer D., Meynet G., \& Maeder A. 1992, A\&AS 96, 269

Smecker-Hane, T.A., Stetson, P.B., Hesser, J.E., and VandenBerg, D.A. 1995, in "From Stars to Galaxies: The Impact of Stellar Physics on Galaxy Evolution", eds. C. Leitherer, U. Fritze-von Alvensleben, and J. Huchra, ASP Conf. Ser., 98, 328

Worthey, G. 1994, ApJS, 95, 107 